\title{
Remerciements aux relecteurs 2020
}

\section{Carmen Renaudeau*}

La revue Cahiers Agricultures remercie tous les relecteurs qui ont bien voulu l'aider à prendre une décision sur la publication des textes et ont fourni aux auteurs de précieux conseils.
Abdelkader Adamou
Jean Josué Albergel
Valérie Angeon
Guilhem Anzalone
Leilla Apithy
Sigrid Aubert
Patrice Autfray
Jean-François Bélières
Olivier Barreteau
Yves Bertheau
Fabio Berti
Cécile Blatrix
Bernard Bodson
Agnès Bonnaud
Ahmed Bouaziz
Alain Bourbouze
Robin Bourgeois
Jeremy Bourgoin
Laurence Boutinot
Thierry Brevault
Dominique Broussal
Laurent Bruckmann
Christopher Bryant
Perrine Burnod
Aurélie Carimentrand
Bruno Cheviron
Mohamed Chikhaoui
Jean-Philippe Choisis
François Claine
Cathy Clermont
Françoise Cognard
Anne-Laure Collard
Claude Compagnone
Christian Corniaux
Kalifa Coulibaly
Pascal Danthu
William's Daré
Manon Dardonville
Hélène David-Benz
Axel Decourtye
Julien Demenois
Luc Descroix

Djiby Dia
Jean-Christophe Diepart
Paul M. Dontsop
Michel Dron
Raphaëlle Ducrot
Patrick Dugué
Patrice Dumas
Bertrand Dumont
Jean François Duranton
Michel Duru
Sandrine Dury
Dorothée Dussy
Guillaume Duteurtre
Mohamed Elloumi
Arilson Favareto
Tionyélé Fayama
Bernard Faye
Laurène Feintrenie
Muriel Figuié
Stéphane Fournier
Franck Galtier
Kouamé Casimir Gboko
Isabelle Goldringer
Sophie Gourguet
Hervé Guibert
Salia Hanafi
Tarik Hartani
Michel Havard
Laure Hossard
Joël Huat
Johann Huguenin
David Hunter
Christian Huyghe
Eric Justes
Alain Karsenty
Renée Koch-Piettre
Cokou Patrice Kpadé
Marcel Kuper
Pierre Labarthe
Luz del Carmen Lagunes Espinoza
Rabah Lahmar
Jacques Lasseur

$\overline{\text { *Auteur de correspondance }}$ : carmen.renaudeau@cirad.fr 
Marie de Lattre-Gasquet

Anne Lauvie

Philippe Lavigne Delville

Mohammed Lazar

Marianne Le Bail

Fabrice Le Bellec

Thomas Le Bourgeois

Jean-François Le Coq

Tristan Le Cotty

Pascal Le Floc'h

Pierre-Yves Le Gal

Crystèle Léauthaud

Caroline Lejars

Guillaume Lescuyer

Jonathan Locqueville

Youenn Lohéac

Dominique Louppe

Jessie Luna

Toufik Madani

Elodie Maître D’Hôtel

Pascal Marnotte

Claire Marsden

Doyle McKey

Philippe Méral

Aziz Merzouk

Gilles Freddy Mialoundama Bakouétila

Etienne Montaigne

Sylvie Morardet

Kevin Morel

Charles-Henri Moulin

Isabelle Mougenot

Paule Moustier

Louis Ndumbe Njie

Oumar Niangado

Serge Nzietchueng

Jean-François Odoux

Joséphine Peigne
Eric Penot

Jean-Louis Pham

Louis-Marie Raboin

Damien Raclot

Bruno Ramamonjisoa

Pierre Ricau

Frédéric Richard

Aude Ripoche

Samira Rousselière

Claire Ruault

François Ruf

Eric Sabourin

Guido Santini

Denis Sautier

Aristide Wendyam Semporé

Georges Serpantié

Mongi Sghaier

Jean-Michel Sorba

Guillaume Soullier

Jean-Michel Sourisseau

Mohamed Taher Sraïri

Geneviève Teil

Marie Thiollet-Scholtus

Jean-Philippe Tonneau

Emmanuel Torquebiau

Anne Toulet

Jean-Marc Touzard

Guy Trébuil

Gilles Trouche

Michel Vaksmann

Elodie Valette

Eric Vall

César Jesús Vázquez-Navarrete

Sibiri Jean Zoundi

Citation de l'article : Renaudeau C. 2021. Remerciements aux relecteurs 2020. Cah. Agric. 30: 11. 